Supplement of Atmos. Chem. Phys. Discuss., 15, 26357-26421, 2015

http://www.atmos-chem-phys-discuss.net/15/26357/2015/

doi:10.5194/acpd-15-26357-2015-supplement

(C) Author(s) 2015. CC Attribution 3.0 License.

(c) (i)

Supplement of

\title{
Shortwave direct radiative effects of above cloud aerosols over global oceans derived from eight years of CALIOP and MODIS observations
}

\section{Z. Zhang et al.}

Correspondence to: Z. Zhang (zhibo.zhang@umbc.edu)

The copyright of individual parts of the supplement might differ from the CC-BY 3.0 licence. 


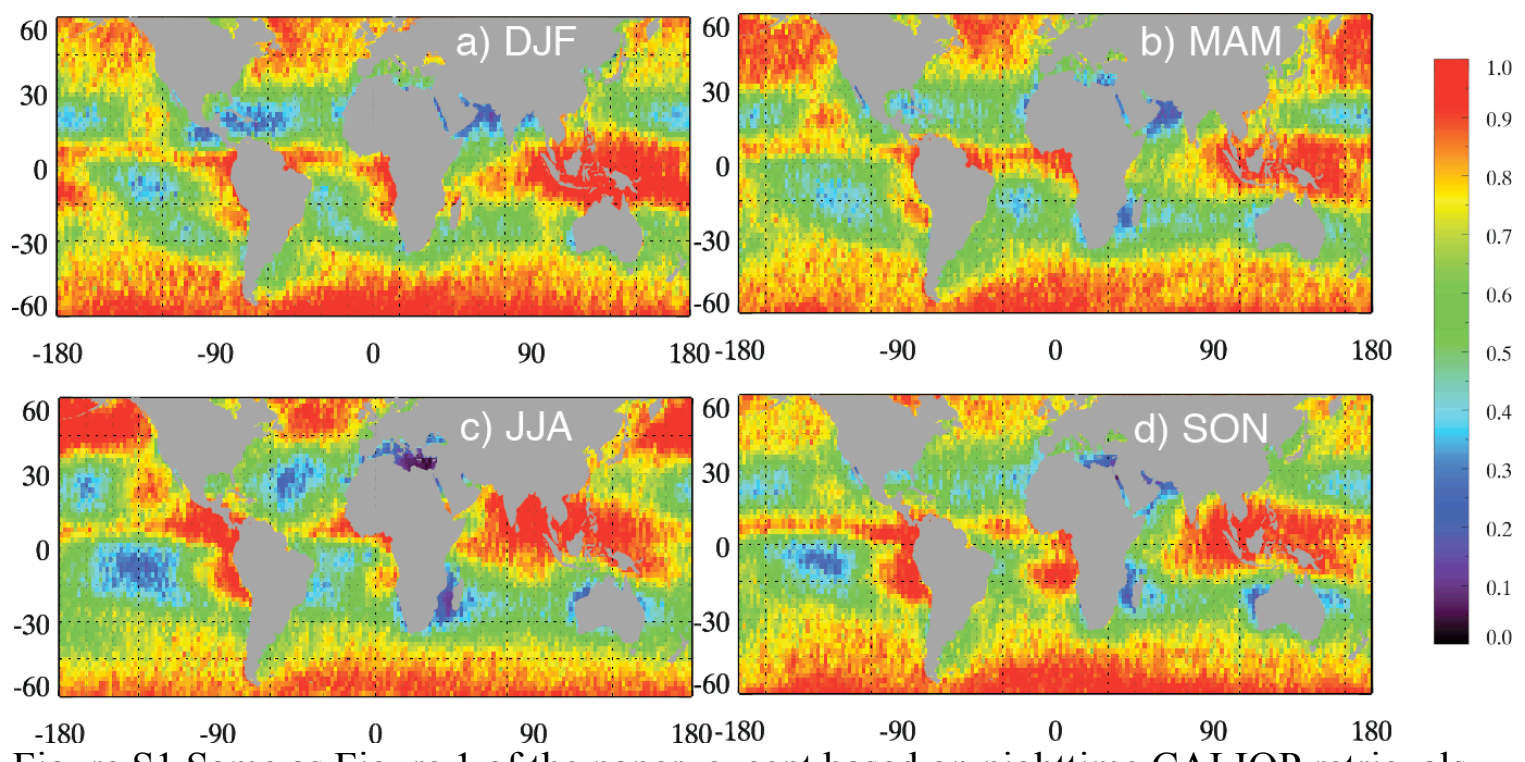

Figure S1 Same as Figure 1 of the paper, except based on nighttime CALIOP retrievals. 

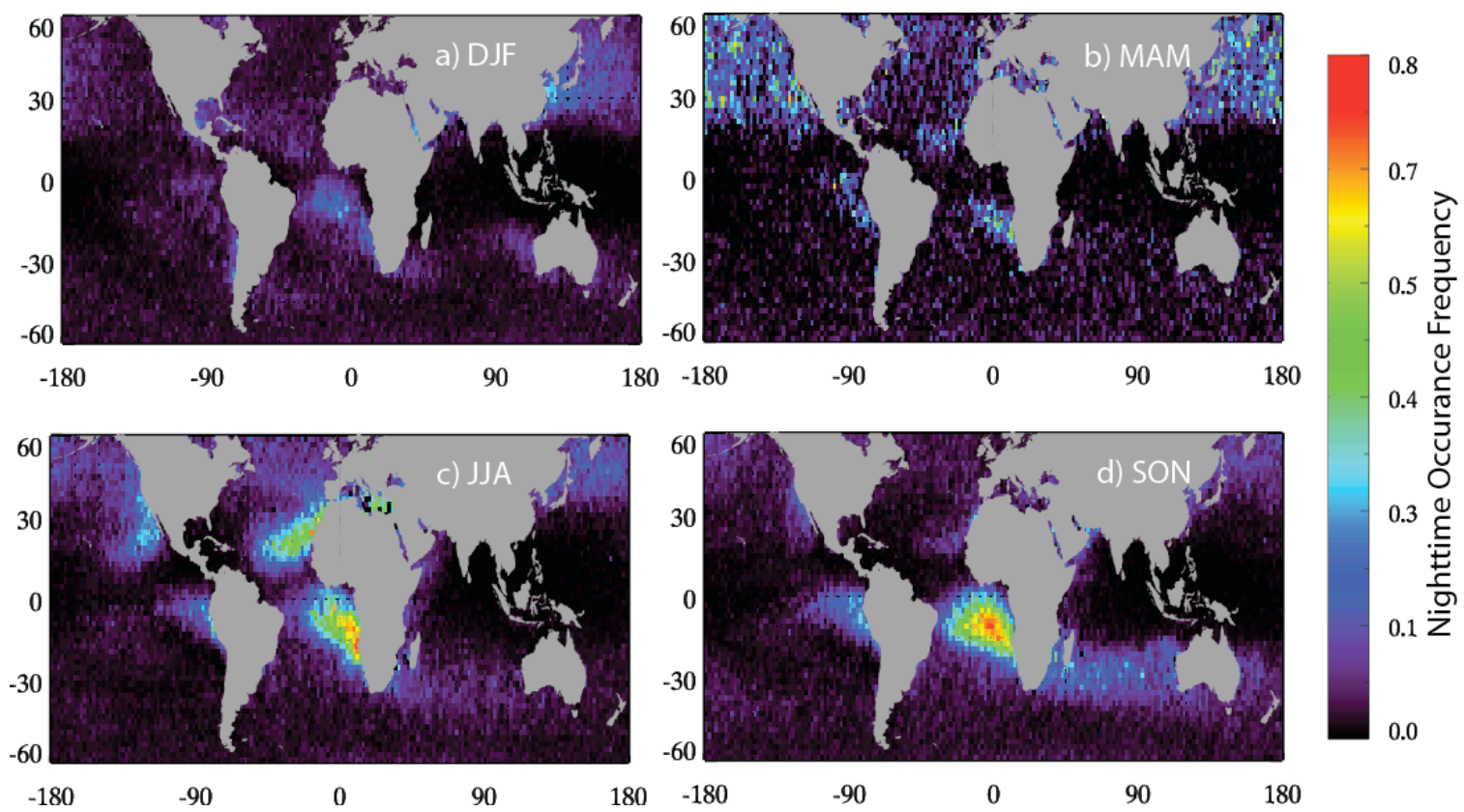

Figure S2 Same as Figure 2 of the paper, except based on nighttime CALIOP retrievals. 
a) Smoke

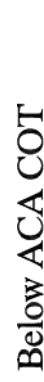

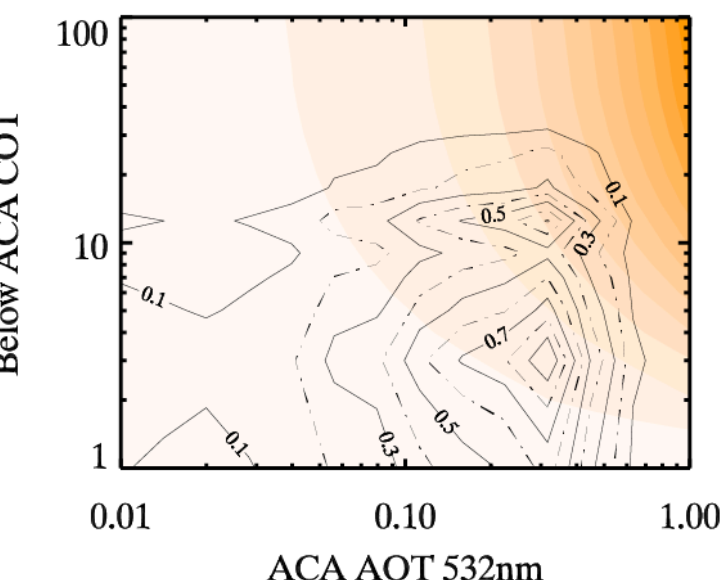

b) Polluted Dust

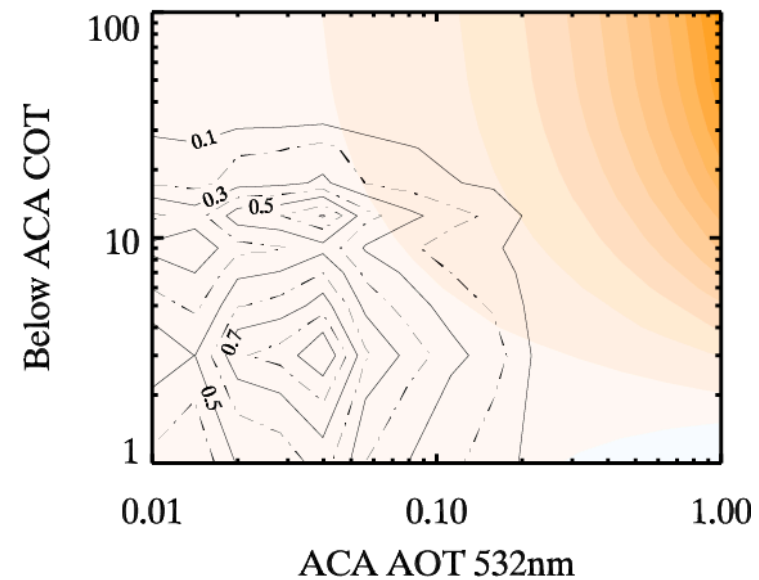

TOA DRE $\left[\mathrm{W} / \mathrm{m}^{2}\right]$

$-100 \quad-75$

$-25$

25

75

100

Figure S3 Same as Figure 11, except based on nighttime CALIOP aerosol retrievals 


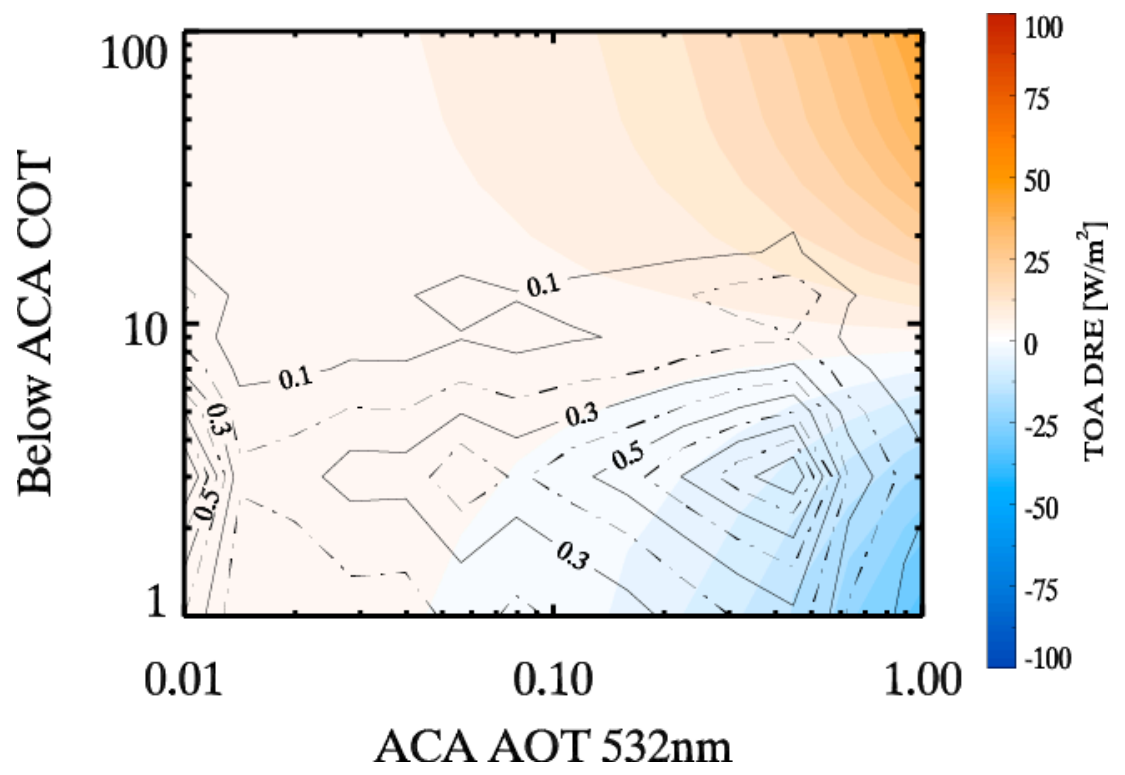

Figure S4 Same as Figure 12a in the paper, except based on nighttime CALIOP retrievals. 

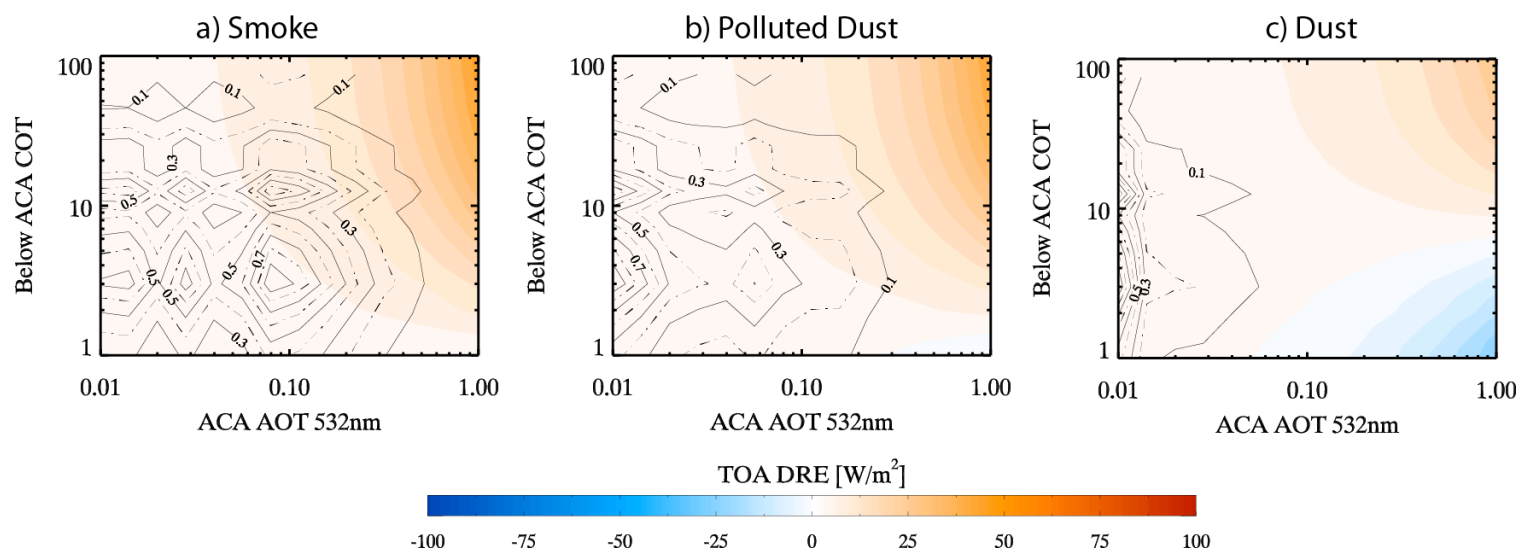

Figure S5 Same as Figure $133^{-75}$ in the paper, except based on nighttime CALIOP retrievals. 
Table S1 Same as the Table 3 in the paper except based on the combination of nighttime CALIOP AOT retrieval and daytime MODIS COT retrieval.

\begin{tabular}{|c|c|c|c|}
\hline Type & $\begin{array}{c}\text { CALIOP smoke+OBS } \\
\text { dust }\end{array}$ & $\begin{array}{c}\text { Haywood } \\
\text { smoke+OBS dust }\end{array}$ & $\begin{array}{c}\text { CALIOP } \\
\text { smoke+OPAC dust }\end{array}$ \\
\hline Smoke & $0.031(0.054)$ & $0.015(0.028)$ & $0.031(0.054)$ \\
& $-0.032(-0.050)$ & $-0.057(-0.092)$ & $-0.032(-0.050)$ \\
& $0.064(0.104)$ & $0.072(0.120)$ & $0.064(0.104)$ \\
\hline Dust & $-0.014(-0.016)$ & $-0.014(-0.016)$ & $-0.004(-0.004)$ \\
& $-0.034(-0.044)$ & $-0.034(-0.044)$ & $-0.042(-0.054)$ \\
& $0.020(0.028)$ & $0.020(0.028)$ & $0.037(0.050)$ \\
& $0.007(0.012)$ & $0.007(0.012)$ & $0.007(0.012)$ \\
& $-0.019(-0.027)$ & $-0.019(-0.027)$ & $-0.019(-0.027)$ \\
& $0.026(0.039)$ & $0.026(0.039)$ & $0.026(0.039)$ \\
\hline
\end{tabular}


Table S2 Same as the Table 4 in the paper except based on the combination of nighttime CALIOP AOT retrieval and daytime MODIS COT retrieval.

\begin{tabular}{|c|c|c|c|}
\hline Region & $\begin{array}{c}\text { CALIOP smoke+OBS } \\
\text { dust }\end{array}$ & $\begin{array}{c}\text { Haywood } \\
\text { smoke+OBS dust }\end{array}$ & $\begin{array}{c}\text { CALIOP } \\
\text { smoke+OPAC dust }\end{array}$ \\
\hline SE Atlantic & $\begin{array}{c}0.38(0.58) \\
-0.48(-0.65) \\
0.84(1.23)\end{array}$ & $\begin{array}{c}0.18(0.31) \\
-0.79(-1.12) \\
0.96(1.42)\end{array}$ & $\begin{array}{c}0.38(0.59) \\
-0.47(-0.66) \\
0.85(1.24)\end{array}$ \\
\hline TNE Atlantic & $\begin{array}{c}-0.32(-0.36) \\
-0.91(-1.05) \\
0.60(0.69)\end{array}$ & $\begin{array}{c}-0.32(-0.37) \\
-0.91(-1.05) \\
0.60(0.69)\end{array}$ & $\begin{array}{c}-0.14(-0.16) \\
-1.07(-1.24) \\
0.94(1.09)\end{array}$ \\
\hline Arabian Sea & $\begin{array}{c}-0.08(-0.08) \\
-0.24(-0.25) \\
0.17(0.17)\end{array}$ & $\begin{array}{c}-0.08(-0.08) \\
-0.25(-0.26) \\
0.17(0.17)\end{array}$ & $\begin{array}{c}-0.04(-0.04) \\
-0.10(-0.27) \\
0.13(0.24)\end{array}$ \\
\hline NW Pacific & $\begin{array}{c}0.15(0.27) \\
-0.14(-0.24) \\
0.29(0.51)\end{array}$ & $\begin{array}{c}0.12(0.23) \\
-0.19(-0.34) \\
0.32(0.56)\end{array}$ & $\begin{array}{c}0.16(0.30) \\
-0.15(-0.25) \\
0.31(0.55)\end{array}$ \\
\hline Global Ocean & $\begin{array}{c}0.02(0.04) \\
-0.09(-0.13) \\
0.11(0.17)\end{array}$ & $\begin{array}{c}0.00(0.02) \\
-0.12(-0.17) \\
0.12(0.19)\end{array}$ & $\begin{array}{c}0.03(0.06) \\
-0.10(-0.14) \\
0.13(0.20)\end{array}$ \\
\hline
\end{tabular}


Table S3 Same as Table S2, except for JJA seasonal mean

\begin{tabular}{|c|c|c|c|}
\hline Region & $\begin{array}{l}\text { CALIOP smoke+OBS } \\
\text { dust }\end{array}$ & $\begin{array}{c}\text { Haywood } \\
\text { smoke+OBS dust }\end{array}$ & $\begin{array}{c}\text { CALIOP } \\
\text { smoke+OPAC dust }\end{array}$ \\
\hline SE Atlantic & $\begin{array}{c}0.50(0.74) \\
-0.67(-0.89) \\
1.17(1.64)\end{array}$ & $\begin{array}{c}0.25(0.42) \\
-1.12(-1.52) \\
1.37(1.94)\end{array}$ & $\begin{array}{c}0.50(0.75) \\
-0.68(-0.89) \\
1.18(1.64)\end{array}$ \\
\hline TNE Atlantic & $\begin{array}{c}-0.99(-1.12) \\
-2.84(-3.22) \\
1.85(2.10)\end{array}$ & $\begin{array}{c}-0.99(-1.13) \\
-2.84(-3.23) \\
1.85(2.10)\end{array}$ & $\begin{array}{c}-0.41(-0.46) \\
-3.36(-3.82) \\
2.96(3.36)\end{array}$ \\
\hline Arabian Sea & $\begin{array}{c}-0.14(-0.14) \\
-0.39(-0.40) \\
0.26(0.26)\end{array}$ & $\begin{array}{c}-0.14(-0.14) \\
-0.39(-0.41) \\
0.26(0.27)\end{array}$ & $\begin{array}{c}-0.08(-0.08) \\
-0.45(-0.47) \\
0.37(0.38)\end{array}$ \\
\hline NW Pacific & $\begin{array}{c}0.22(0.41) \\
-0.18(-0.30) \\
0.40(0.71)\end{array}$ & $\begin{array}{c}0.18(0.33) \\
-0.27(-0.48) \\
0.45(0.81)\end{array}$ & $\begin{array}{c}0.23(0.42) \\
-0.18(-0.30) \\
0.41(0.73)\end{array}$ \\
\hline Global Ocean & $\begin{array}{c}0.01(0.04) \\
-0.16(-0.20) \\
0.17(0.24)\end{array}$ & $\begin{array}{c}-0.01(0.02) \\
-0.19(-0.17) \\
0.18(0.19)\end{array}$ & $\begin{array}{c}0.03(0.06) \\
-0.18(-0.22) \\
0.21(0.28)\end{array}$ \\
\hline
\end{tabular}

